\title{
Shape estimation for Košice, Almahata Sitta and Bassikounou meteoroids
}

\author{
Vladimir Vinnikov ${ }^{1}$, Maria Gritsevich ${ }^{1,2,3}$ and Leonid Turchak ${ }^{1}$ \\ ${ }^{1}$ Dorodnicyn Computing Centre of the Russian Academy of Sciences, Department of \\ Computational Physics, Vavilova ul. 40, 119333, Moscow, Russia \\ email: vvinnikov@list.ru \\ ${ }^{2}$ Finnish Geodetic Institute, P.O. Box 15, FI-02431 Masala, Finland; \\ email: maria.gritsevich@fgi.fi \\ ${ }^{3}$ Institute of Physics and Technology, Ural Federal University, Mira ul. 21, 620002, \\ Ekaterinburg, Russia
}

\begin{abstract}
This paper is concerned with a meteoroid shape estimation technique based on statistical laws of distribution for fragment masses. The idea is derived from the experiments that show that brittle fracturing produces multiple fragments of size lesser than or equal to the least dimension of the body. The number of fragments depends on fragment masses as a power law with exponential cutoff. The scaling exponent essentially indicates the initial form of the fragmented body. We apply the technique of scaling analysis to the empirical data on the mass distributions for Košice, Almahata Sitta and Bassikounou meteorites.
\end{abstract}

Keywords. Methods: data analysis, methods: statistical, Shape estimation, scaling law, meteoroid fragmentation, mass distribution.

\section{Introduction}

The estimation of initial meteoroid shape is crucial, since the meteoroid pre-entry mass, terminal meteorite mass, and fireball luminosity are proportional to the pre-entry shape factor of the meteoroid to the power of 3 (Gritsevich \& Koschny 2011). However, its reconstruction is not straightforward. Unlike a classical puzzle, the meteorite fragments found within one fall do not match each other since not all body splinters reach the Earth's surface, and those that do suffer from melting and ablation, which smooth distinct crack surfaces and round sharp corners. Fortunately, in many cases the ablation is considered to be weak after the major fragmentation occurred (Spurny et al. 2003; Gritsevich 2008) and thus the resulting mass distribution does not change significantly (Oddershede et al. 1998). Moreover, the brittle shattering has fractal properties similar to many other natural phenomena (e.g. Lang \& Franaszczuk 1986; Nagahama 1993). This self-similarity for scaling mass sequences is described by power law statistical expressions (Oddershede et al. 1993; Amitrano 2012). Simply speaking, it means that any observed limited subset of small splinter masses does not give any information about their relative location in the overall distribution. This subset can be placed anywhere on the probability density curve, resulting only in scale change of the total mass. The same feature is exhibited by more classical fractals, such as clouds or mountain rocks. It is theoretically impossible to deduce the absolute size of fractal objects while watching their parts without comparison to surroundings. 


\section{Shape estimation}

The experiments on brittle fracturing demonstrate that the resulting multiple fragments have the size lesser than or equal to the least dimension of the body (Oddershede et al. 1993). The shape-estimation technique is based on the idea that such pieces obey the power law with scaling exponent $\beta_{0}$ and exponential cutoff: $F_{c}(m)=$ $C \cdot m^{-\beta_{0}} \cdot \exp \left(-m / m_{U}\right)$, where $C=\left(\beta_{0}-1\right) \cdot m_{L}^{\beta_{0}-1}$ is a normalization constant, $m_{U}>$ $m_{L}$ is an upper cutoff fragment mass and $m_{L}$ is an arbitrary lower mass limit, acting as an additional constraint for undersampled tiny unrecoverable fragments. These mass constraints are also among the sought parameters. The meteorite sample consists of $N$ fragments ranging in mass from $m_{0}$ to $m_{N-1}$ presented in ascending order. If two or more fragments yield equal tabular masses, we add a small value corresponding to the fragment mass measurements error (for example, $0.001 \mathrm{~g}$ ) to one of them, and move the masses apart. Since the density distribution $n(m)$ of fragment masses $\left\{m_{i}\right\}_{i=1}^{N}$ is discrete, we convert it to the piecewise complementary cumulative distribution function (CCDF) $N^{*}(m)$ as suggested in (Oddershede et al. 1998): $N^{*}(m)=\frac{1}{m} \int_{m}^{\infty} n(x) d x$, where $m \geqslant m_{L}, n(m)=\sum_{i=1}^{N} \delta\left(m-m_{i}\right)$ and $\delta$ is the Dirac delta function.

In order to match $F_{c}(m)$ to $N^{*}(m)$ we conduct a normalization procedure, equaling their values at the point $m_{L}$. For simplicity, we also assume that the lower bound $m_{L}$ coincides with the mass of the fragments $m_{j}$, i.e. $n_{L}=j, m_{L}=m j$. Thus the normalization constant $C$ can be expressed as follows: $C=\left(m_{j}\right)^{\beta_{0}-1} \cdot \exp \left(-m_{j} / m_{U}\right) \cdot(N-j)$. The CCDF becomes: $F_{c}(m)=\frac{N-j}{m_{j}} \cdot\left(m / m_{j}\right)^{-\beta_{0}} \cdot \exp \left(-\left(m-m_{j}\right) / m_{U}\right)$. We note here that the scaling exponent $\beta_{0}$ is non-dimensional and has no dependence on the dimensions of $N^{*}(m)$ and $F_{c}(m)$.

Next, the least-squares method is applied to minimize the expression:

$S\left(\beta_{0}, j \cdot m_{U}\right)=\sum_{i=j}^{N}\left[F_{c}\left(m_{i}\right)-(N-j) / m_{j}\right]^{2}$. We use the Newtons method to get $\beta_{0}$ and $m_{U}$ for the valid range of $m_{L}$. Once $\beta_{0}$ is obtained, we can estimate dimensionless shape parameter $d$ and size proportions $a_{x}, a_{y}, a_{z}$ from the empirical equations $0.13 d^{2}-0.21 d+\left(1.1-\beta_{0}\right)=0$ and $d=1+2\left(a_{x} \cdot a_{y}+a_{y} \cdot a_{x}+a_{z} \cdot a_{x}\right)\left(a_{x}^{2}+a_{y}^{2}+a_{z}^{2}\right)^{-1}$. We emphasize that these equations and relations do have the area of applicability limited to homogeneous brittle solids that are not prestrained. It is known that prestrained materials (e.g. tempered glass) while being brittle do not follow power law mass distribution under fragmentation.

\section{Results}

We use the described technique on the set of mass distributions, corresponding to well known meteorite showers with large number of recovered fragments. Here we present results based on 3 cases, namely: Košice, Bassikounou and Almahata Sitta. The respective data can be found in (Buhl \& Baermann 2007; Shaddad et al. 2010; Gritsevich et al. 2014). All mass distributions exhibit common features of undersampling for small fragments and exponential cutoff for a finite size effect. The estimated values of scaling exponent $\beta_{0}$, lower and upper constraints $m_{L}, m_{U}$ and dimensionless shape parameter $d$ are presented in the table 1 . The corresponding plots are provided in figures $1,2,3$.

The most interesting mass distribution is related to the Almahata Sitta meteorite shower (Shaddad et al. 2010). The plot (Fig.3) demonstrates two distinctive linear subranges. The first subrange doubtfully can be attributed only to the undersampling effect. The number of fragments within it is large, and their masses obey the scaling law with $\beta_{0}$ equal to 1.0. It is possible to construct the composite power law which fits both 
subranges. However the coefficients of the empirical equation imply that the scaling exponent $\beta_{0}$ should be greater than 1.1, if the roots $d$ are expected to be real value. We anticipate that the Almahata Sitta distribution is caused by the independently fragmented meteoroids with different properties. This problem is a subject of the following study.

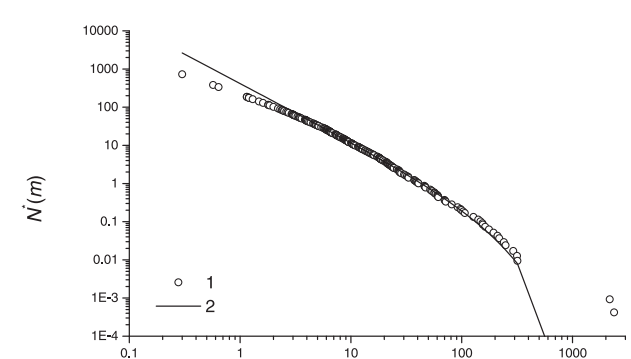

Figure 1: Complementary cumulative number of fragments $N^{*}(m)$ vs $m .1$ Observed data, 2 - Power law distribution with exponential cutoff. Košice meteorite.

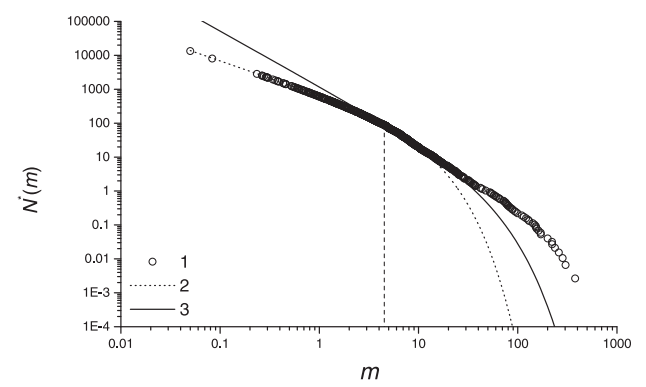

Figure 3: Complementary cumulative number of fragments $N^{*}(m)$ vs $m .1$ Observed data, 2,3 - Power law distributions with exponential cutoff. Almahata Sitta meteorite.

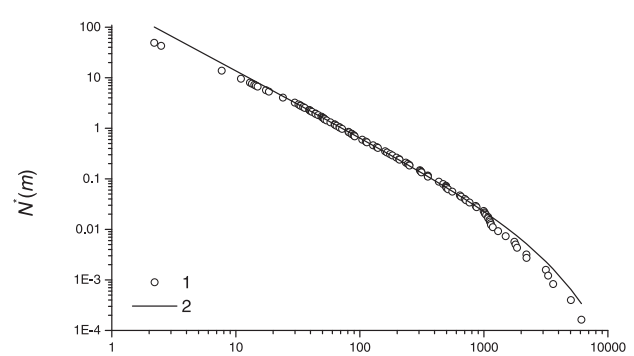

Figure 2: Complementary cumulative number of fragments $N^{*}(m)$ vs $m .1$ - Observed data, 2 - Power law distribution with exponential cutoff. Bassikounou meteorite.

Table 1: The derived shape parameters for the selected meteoroids

\begin{tabular}{lllll}
\hline Meteoroid & $\beta_{0}$ & $m_{L},[\mathrm{~g}]$ & $m_{U},[\mathrm{~g}]$ & $d$ \\
\hline Košice & 1.53 & 5.64 & 155.17 & 2.8 \\
\hline Bassikounou & 1.32 & 29.9 & 2839.42 & 2.34 \\
\hline Almahata Sitta & 1.0 & 0.6 & 7.84 & - \\
& 1.64 & 4.51 & 31.97 & 3.0 \\
\hline
\end{tabular}

\section{References}

Amitrano, D 2012, Eur. Phys. J. Spec. Top., 205, 199

Buhl, S. \& Baermann, M. 2007, Niger Meteorite Recon, www.niger-meteorite-recon.de

Gritsevich, M. 2008, Sol. Syst. Res., 42, 372

Gritsevich, M. \& Koschny, D. 2011, Icarus, 212, 877

Gritsevich, M., Vinnikov, V., Kohout, T., Tóth, J., Peltoniemi, J., Turchak, L., \& Virtanen, J. 2014, Meteorit. Planet. Sci., 49, 328

Lang, B. \& Franaszczuk, K. 1986, Meteoritics, 21, 425

Nagahama, H. 1993, Int. J. Rock Mech. Min. Sci. \& Geomech. Abstr., 30, 469

Oddershede, L., Dimon, P., \& Bohr, J. 1993, Phys. Rev. Lett., 71, 3107

Oddershede, L., Meibom, A., \& Bohr, J. 1998, Europhys. Lett, 43, 598

Shaddad, M. H., Jenniskens, P., Numan, D., Kudoda, A. M., Elsir, S., Riyad, I. F., Ali, A. E., Alameen, M., Alameen, N. M., Eid, O., Osman, A. T., Abubaker, M. I., Yousif, M., Chesley, S. R., Chodas, P. W., Albers J., Edwards, W. N., Brown, P. G., Kuiper, J., \& Friedrich, J. M. 2010, Meteorit. Planet. Sci., 45, 1557

Spurny, P., Oberst, J., \& Heinlein, D. 2003, Nature, 423, 151 